\title{
The effective use of cardiac enzymes and electro- cardiograms in the diagnosis of acute myocardial infarction in the elderly
}

\author{
R. Gama ${ }^{1}$, D.G. Swain ${ }^{2}$, P.G. Nightingale ${ }^{1}$ and B.M. Buckley ${ }^{3}$ \\ Departments of ${ }^{1}$ Clinical Chemistry, Wolfson Research Laboratories, Queen Elizabeth Medical Centre, \\ Edgbaston, Birmingham B15 2TH, ${ }^{2}$ Geriatric Medicine and ${ }^{3}$ Clinical Chemistry, Sandwell District General \\ Hospital, West Bromwich, Birmingham, UK.
}

\begin{abstract}
Summary: The diagnosis of acute myocardial infarction (AMI) in the elderly is difficult and often depends on the results of investigations. In a 3-month prospective study, 270 patients admitted to an acute geriatric unit were studied to determine the most effective diagnostic strategy for the diagnosis of AMI, and to assess the value of screening acute geriatric admissions for AMI.

Patients were assessed clinically and investigated with serial electrocardiograms and measurements of serum creatine kinase (CK), aspartate aminotransferase (AST), and lactate dehydrogenase activities on three consecutive days after admission.

Measurement of serum activity of CK and AST had a diagnostic sensitivity of $100 \%$ and specificity of $\mathbf{8 6 . 8} \%$ for AMI. This was the optimum combination of cardiac enzymes in the diagnosis of AMI. Although electrocardiograms on the first two days of admission had a low diagnostic sensitivity (33.3\%) their usefulness was their high positive predictive value $(100 \%)$ when characteristic of an AMI.

AMI was considered in the differential diagnosis of 79 patients (29\%). All 25 patients ( $9 \%$ ) who had an AMI were in this group, and therefore screening all geriatric admissions for AMI is not clinically justified.
\end{abstract}

\section{Introduction}

Acute myocardial infarction (AMI) is a common cause for hospital admission to acute geriatric units. The diagnosis of AMI is difficult, ${ }^{1}$ and especially in the elderly who may present silently or with non-specific clinical features. ${ }^{2,3}$ In addition, the prognosis of AMI worsens with increasing age. ${ }^{4}$ These factors have led to the suggestion that ill elderly patients should be screened for AMI. ${ }^{3}$

There is no consensus on biochemical tests nor on numbers of electrocardiograms (ECG) required in the diagnosis of AMI. The choice of biochemical test(s) and how often they should be measured varies not only between but also within countries. $^{5-7}$

The aims of this prospective study were firstly to optimize a strategy of serial electrocardiograms (ECG) and serial measurements of creatine kinase (CK), aspartate aminotransferase (AST) and lactate dehydrogenase (LDH) activities in the investigation of AMI in the elderly, and secondly to assess the value of screening acute geriatric admissions for AMI.

Correspondence: R. Gama M.R.C.P.

Accepted: 22 November 1989

\section{Methods and materials}

All patients admitted to the acute geriatric unit between May and July 1988 entered the study. Each patient was assessed clinically and investigated with serial 12-lead ECGs and measurement of serum activities of CK, AST and LDH on days 1 , 2 and 3 of admission.

CK, AST and LDH activities were measured with standard techniques using a Cobas FARA (Roche Products, Welwyn Garden City, Herts.) with reagents supplied by Roche Products and BCL (Lewes, Sussex). Reference ranges $(95 \%$ confidence intervals) for CK, AST and LDH were $17-188 \mathrm{U} / 1,12-32 \mathrm{U} / 1$ and $226-616 \mathrm{U} / 1$ respectively, and were based on 60 fit residents of local rest homes aged $83.1 \pm 5.8$ years (mean \pm s.d.), of whom 31 were female.

Clinical events were classified independently by three physicians into three groups using previously described criteria. ${ }^{1}$ These were: (i) Definite AMI: history sufficient to warrant hospital admission for AMI plus at least either an ECG characteristic of an AMI or elevation of one or more cardiac enzyme activities to greater than twice the upper limit of normal. (ii) Possible AMI: history as above with an 
abnormal ECG not characteristic of an AMI and elevation of one or more cardiac enzyme activities but to less than twice the upper limit of normal. (iii) No AMI: none of the above.

Various combinations of cardiac enzymes and ECGs were evaluated for sensitivity, specificity, efficiency and predictive values for the diagnosis of definite AMI. ${ }^{8}$ Biochemical tests and ECG were costed as previously described.

\section{Results}

There were 279 admissions to the acute geriatric unit over the 3 month period studied. Nine patients were excluded because of a failure to have either ECGs or cardiac enzymes done leaving 270 patients aged 81.2 \pm 5.7 years (mean \pm s.d.) of whom 179 were female. Five patients had a further clinical event whilst in hospital, of which AMI was considered in the differential diagnosis. Thus 275 clinical events were evaluated.

There were 25 Definite, 16 Possible and 234 No AMIs. AMI was considered in the differential diagnosis of $79(29 \%)$ clinical events. All 25 cases eventually diagnosed as Definite AMI fell into this group. The clinical presentations of Definite AMI were chest pain $(44 \%)$, dyspnoea $(28 \%)$, weakness $(12 \%)$, syncope $(8 \%)$, epigastric pain $(8 \%)$, sweats $(8 \%)$, confusion $(4 \%)$, nausea $(4 \%)$ and transient ischaemic attack (4\%). There were no silent AMIs (absence of symptoms attributable to AMI but a diagnostic ECG and/or elevation of one or more cardiac enzymes to greater than twice the upper limit of normal).

Diagnostic sensitivity, specificity, efficiency and predictive values of the ECG and the various cardiac enzyme combinations for Definite AMI are shown in Table I. Diagnostic sensitivity for individual enzymes increased with the number of serial measurements; of these, CK on three days had the highest sensitivity (84\%). Serial combinations of enzymes had higher sensitivities than individual enzymes measured serially. Maximal sensitivity $(100 \%)$ with the least number of tests was achieved by measuring CK and AST on the first two days following admission. Although diagnostic sensitivity, specificity, efficiency and predictive values depend on the prevalence of AMI in the population studied, these values were similar when applied to the 79 cases in whom AMI was considered in the differential diagnosis.

The sensitivity of ECGs in the diagnosis of AMI was low when compared to cardiac enzymes. An ECG characteristic of a Definite AMI was always associated with cardiac enzyme levels elevated to greater than twice the upper limit of normal. The reverse, however, did not apply. Thirty-four cases $(15 \%)$ in the No AMI category had at least one 'cardiac enzyme' activity elevated to greater than twice the upper limit of the reference range durins the first three days after admission.

The revenue and variable costs of serial ECG\$ and measurement of serum CK, AST and LDH activities on the first three days of admission were $£ 21.48$ and $£ 2.13$ respectively, and of ECGs and

Table I Diagnostic sensitivity, specificity, efficiency and predictive values for Definite AMI of ECG and cardiac enzymes in various combinations

\begin{tabular}{|c|c|c|c|c|c|c|}
\hline \multirow[b]{2}{*}{ Tests } & \multirow[b]{2}{*}{ Days } & \multicolumn{2}{|c|}{ Diagnostic } & \multirow[b]{2}{*}{$\begin{array}{c}\text { Efficiency } \\
\%\end{array}$} & \multicolumn{2}{|c|}{ Predictive values } \\
\hline & & $\begin{array}{c}\text { Sensitivity } \\
\%\end{array}$ & $\underset{\%}{\text { Specificity }}$ & & $\begin{array}{l}+v e \\
\text { result } \\
\%\end{array}$ & $\begin{array}{c}-v e \\
\text { result } \\
\%\end{array}$ \\
\hline CK, AST, LDH & $1,2,3$ & 100 & 84.0 & 85.5 & 38.4 & 100 \\
\hline CK, AST, LDH & 1,2 & 100 & 86.4 & 87.6 & 42.3 & 100 \\
\hline CK, AST, LDH & 1 & 64.0 & 91.6 & 89.0 & 43.2 & 96.2 \\
\hline CK, AST & $1,2,3$ & 100 & 85.6 & 86.9 & 40.9 & 100 \\
\hline CK, AST & 1,2 & 100 & 86.8 & 88.0 & 43.1 & 100 \\
\hline CK, AST & 1 & 64.0 & 91.6 & 89.1 & 43.2 & 96.2 \\
\hline CK & $1,2,3$ & 84.0 & 92.0 & 91.3 & 51.2 & 98.2 \\
\hline CK & 1,2 & 76.0 & 92.4 & 90.9 & 50.0 & 97.4 \\
\hline CK & 1 & 52.0 & 94.8 & 90.9 & 50.0 & 95.2 \\
\hline AST & $1,2,3$ & 80.0 & 91.6 & 90.5 & 48.7 & 97.5 \\
\hline AST & 1,2 & 80.0 & 93.2 & 92.0 & 52.6 & 97.9 \\
\hline AST & 1 & 52.0 & 96.8 & 92.7 & 61.9 & 95.2 \\
\hline LDH & $1,2,3$ & 36.0 & 96.4 & 90.9 & 50.0 & 92.1 \\
\hline LDH & 1,2 & 25.0 & 97.2 & 90.6 & 46.2 & 92.7 \\
\hline LDH & 1 & 16.0 & 98.4 & 90.9 & 50.0 & 92.1 \\
\hline ECG & $1,2,3$ & 44.0 & 100 & 94.9 & 100 & 94.7 \\
\hline ECG & 1,2 & 33.3 & 100 & 93.8 & 100 & 92.9 \\
\hline ECG & 1 & 16.6 & 100 & 92.3 & 100 & 92.3 \\
\hline
\end{tabular}


measurement of serum CK and AST activities on the first two days of admission $£ 13.16$ and $£ 1.26$ respectively.

\section{Discussion}

In view of the severity of AMI in the elderly, optimum sensitivity (no AMIs missed) is more important than specificity (fewest false positives) or efficiency (\% of results which are correct). In this study, measurement of serum CK and AST on the first two days of admission had a sensitivity of $100 \%$ and specificity of $86.8 \%$ for the diagnosis of a Definite AMI. This was the best combination of cardiac enzymes to achieve maximum sensitivity with the minimum number of tests.

The sensitivity of ECGs for the diagnosis of AMI was low when compared to biochemical tests. The value of the ECG was its high predictive value for a positive result, that is a diagnostic ECG was always due to a Definite AMI. In contrast, although 'cardiac enzymes' are considered to be diagnostic tests a significant proportion (15\%) of cases who did not have a Definite or Possible AMI had 'diagnostically' elevated cardiac enzyme activities. Care must, therefore, be taken in attributing elevated cardiac enzyme activity to myocardial injury when there is little clinical or ECG evidence to support this.

In this unit, ECGs and measurement of serum CK and AST activities on the first two days of admission has replaced our previous strategy of serial ECGs and measuring serum CK, AST and LDH activities on the first three days of admission.

What are the net savings in reducing the number of biochemistry tests from 9 to 4 and ECGs from 3 to 2 ? Laboratory revenue costs may be divided into those which are fixed and independent of workload and those which are variable and directly propor-

\section{References}

1. Rowley, J.M. \& Hampton, J.R. Diagnostic criteria for myocardial infarction. Br J Hosp Med 1981, 26: 253-258.

2. Editorial. Presentation of myocardial infarction in the elderly. Lancet 1986, ii: 1077-1078.

3. Bayer, A.J., Chadha, J.S., Farag, R.R. \& Pathy, M.S.J. Changing presentation of myocardial infarction with increasing old age. J Am Geriatr Soc 1986, 34: 263-266.

4. Williams, B.O., Begg, T.B., Semple, T. et al. The elderly in a coronary care unit. $\mathrm{Br} \mathrm{Med} J$ 1976, ii: 451-453.

5. Broughton, P.M.G. \& Worthington, D.J. Laboratories respond differently to the same clinical request. Ann Clin Biochem 1989, 26: 119-121.

6. Henderson, A.R. \& Gardner, M.D. Patients with suspected myocardial infarction: their test request patterns for clinical biochemistry in a British and Canadian cardiac care unit. $J$ Clin Pathol 1986, 39: 803-807. tional to the numbers of tests done. ${ }^{10}$ The net savings in changing strategies will, therefore, be those of consumable costs (variable expenditure) equivalent to only $£ 0.87$ per patient.

In this study $9 \%$ of patients had a Definite AMI and $6 \%$ a Possible AMI. Over half the presentations of Definite AMI were atypical. The physicians were aware of this in that AMI was considered a possibility in one third of admissions. This awareness and absence of silent AMIs suggests that screening all geriatric admissions is not justified and that investigation should be guided by clinical factors. Furthermore, screening all admissions with ECGs and measurement of serum CK and AST activities on the first two days of admission would unnecessarily cost (variable expenditure) an extra $£ 987$ per annum.

Although the cost savings are small there are other important benefits in reducing the number of unnecessary tests. Fewer false positive results would be expected to lead to a reduction in further perhaps unnecessary investigations. One less venepuncture for the patient and more rapid diagnosis represents an improvement in the quality of patient care. The time saved by the doctor, and the laboratory and ECG technicians could be redeployed to other areas of clinical or laboratory activity.

This study was undertaken not to evaluate new tests but to optimize our current diagnostic tools with the classical approach popularized by Galen and Gambino ${ }^{8}$ and it illustrates that increased diagnostic effectiveness, cost-effectiveness and improved patient care can be complementary.

\section{Acknowledgement}

R.G. and P.G.N. acknowledge financial support from the DoH.

7. Skendel, L.P. How many different tests are sufficient for the diagnosis of acute myocardial injury? Clin Chem 1988, 34: 793-794.

8. Galen, R.S. \& Gambino, S.R. Beyond Normality: The Predictive Value and Efficiency of Medical Diagnoses. John Wiley \& Sons, New York, London, Sidney, Ontario, 1975 , pp. 9-14, 30-39.

9. Broughton, P.M.G. \& Hogan, T.C. A new approach to the costing of clinical laboratory tests. Ann Clin Biochem 1981, 18: $330-342$.

10. Pearson, J.R. Romfh, P.C., Habib, J.M. \& Frieling, M.J. The flexible budget process - a tool for cost containment. Am J Clin Pathol 1985, 84: 202-208. 